\title{
Human Parasite Eggs Image Characteristics Extraction and Recognition
}

\section{Technology Research}

\author{
Chunjuan Fang \\ School of Nursing,Jiangxi University of Technology
}

Keywords: Digital image processing; Parasitology; Mode recognition; Texture features

\begin{abstract}
In terms of theory, any issues related to morphological analysis and test can be settled by digital image process technology, but due to the irregular morphological structures of the parasite eggs in human body, spatial positions and too many impurities of the samples, there remains little research on the digital image technology in the field of parasitology and the parasite eggs cannot be recognized by automatic instrument as it does to blood cell, therefore, for the long term, it can be only observed and distinguished by naked eyes under the microscope. Because of the lack of objective, stable and accurate quality in artificial recognition, recognition ability tends to be subject to the experience and status of the testers; what's more, the images, data and results of the test samples are not convenient for storage, reproduction and query, which have left artificial recognition unable to meet the demands of modern hospital informatization. On such basis, the contents of digital image process and model recognition technology, and the images of 10 common parasite eggs in human body have been researched in the thesis. By use of VISua1C++6.0 system development tool and digital process technology, 24 true color parasite eggs collected by microscopic camera have been pretreated to extract the information on their morphology, color and texture.
\end{abstract}

\section{Introduction}

From the far X-ray diagonasis to modern MRI, medical image process is always associated with radiodiagnosis, filmstrip and analogue image. It is generally believed in the industry that medical image shall be ascribed to Roentgen. Microscope is the first invention to make human able to explore the mysterious microworld, while it is after the combination of camera and microscope that the concept of medical microscopic image was born, before the digital image technology achieves adequate development, medical image has been described relying on graphic display and word description, and subject to artificial process by visual observation under and brain analysis.

Human parasitic disease remains the important disease threatening people's health in our country. Parasite eggs recognition is the major method for common parasitic disease diagnosis. The previous recognition of parasite eggs is mainly conducted with naked eyes under microscope after artificial smearing process for human excreta and secreta. The practice is not only burdensome, inadequately objective and accurate, and the recognition error is dependent on the experience and status of the testers, and also it is not convenient to store, reproduce and query the images, data and results of test sample, therefore, it is unable to meet the informatization demands of modern medical service.

In terms of theory, any issues related to morphological analysis and test can be settled by digital image process technology, but due to the irregular morphological structures of the parasite eggs in human body, spatial positions and too many impurities of the samples, parasite eggs cannot be 
recognized by the current automatic analyzer by means of electrical impedance principle as it does to blood cell. Digital image technology remains at the initial research stage, rarely applied and researched in the parasitology.

Judging from the research data retrieved at present, the common problem in the research on digital image automatic recognition of parasite eggs at home and aboard is the lack of practicality and the consideration for the development in the trend of automatic instrumentation recognition to make it more acceptable to clinical application, therefore, the research is made almost purely for sake of research. The major recognition system can recognize a small variety of parasite eggs, usually only limited to laboratory research of several or certain parasite egg or adult parasite. The research with a narrow scope of application does not produce a high practice value in clinical operation.

Based on the theoretic methods of the treatise and the current research documents on digital image process, it has been discovered in the thesis that the irregular morphological structures, spatial positions and impure environment are the major reason for the relatively slow progress of automatic recognition, after the analysis on the research status of digital image recognition. To grasp the diversified microscopic structures of the living forms, the characteristics of most representative species must be fully researched; and by analyzing characteristic differences of various parasite eggs and the extraction calculation, referring, transplanting and transforming the processing methods in other similar image recognition research, a whole set of integrated image recognition experiment system must be prepared which is suitable for human common parasite eggs image characters with simple procedure and fast processing speed.

\section{Parasite egg image smoothing algorithm analysis}

smoothing algorithm aims to contain noise and improve image quality. Digital image smoothing technology can be divided into two types. One of the types is for local process to get a smooth image by correcting the whole or the large part of the noise image, such as using Wiener filtering and the least squares filtering in transform domain. These technologies require knowing the statistic model of the signals and noise, however, people are ignorant or impossible to use simple random process to accurately describe the statistical model, and what's more, the technologies are not suitable for the thesis for their large calculation burden. The other digital image smoothing technology uses local operator for noise image, which means while smoothing process for a pixel, the calculation only covers its local small neighborhood domain. The advantage of the technology is the great calculation efficiency and parallel process for multiple pixels. This smoothing technology for local neighborhood is right adopted in the thesis. On such basis, the whole pretreatment is finished by use of local neighborhood domain calculation.

The common method for neighborhood domain calculation is template convolution operation. To ave calculation time, $3 \times 3$ template is used in the thesis, and at the same time, among spatial filtering and frequency domain filtering with intensifying images, the latter left out of use for its large calculation burden and memory occupation.

The common spatial smoothing filter includes linear smoothing filter and mid-value filter, based on the image characteristics in the topic, the thesis mainly focuses on linear smoothing filter, instead of the mid-value filter that mainly suits pulse noise, scanning noise with slow calculation speed. 


$$
\begin{gathered}
\text { (1) } \frac{1}{4}\left[\begin{array}{lll}
0 & 1 & 0 \\
1 & 1 & 1 \\
0 & 1 & 0
\end{array}\right] \quad \text { (2) } \frac{1}{8}\left[\begin{array}{lll}
1 & 1 & 1 \\
1 & 0 & 1 \\
1 & 1 & 1
\end{array}\right] \\
\text { (3) } \frac{1}{9}\left[\begin{array}{lll}
1 & 1 & 1 \\
1 & 1 & 1 \\
1 & 1 & 1
\end{array}\right] \text { (4) } \frac{1}{10}\left[\begin{array}{lll}
1 & 1 & 1 \\
1 & 2 & 1 \\
1 & 1 & 1
\end{array}\right] \text { (5) } \frac{1}{16}\left[\begin{array}{lll}
1 & 2 & 1 \\
2 & 4 & 2 \\
1 & 2 & 1
\end{array}\right]
\end{gathered}
$$

Almost all the books about image process are silent about the detailed functions and differences of these templates, except the introduction of their functions of saltation removal point or the blurring effects to remove the small details. Actually, only the careful analysis on the template central point and the gray average of the adjacent pixels can help us notice that the weights of the central, horizontal and vertical pixel points are the key to deciding the noise removal and blurring effects.

\section{Parasite eggs image sharpening analysis}

Image sharpening aims to strengthen the boundary and contour of the scene in the image. Image SNR (Signal to Noise Ratio) is intensified after image smoothing noise reduction, but the boundary becomes blurred. The sharpening process is to get a bigger gray scale contrast, because the boundary and the contour are both at the part of saltation gray scale. It is necessary for the image recognition procedure requiring boundary test and perimeter and size measurement.

There are two common differential sharpening methods-gradient sharpening and Laplace sharpening, and the former implies using gradient for differential operation.

For image $\mathrm{f}(\mathrm{x}, \mathrm{y})$, the range differential expression of its gradient can be simplified as the gradient differential formula as below:

$$
\left[(, G) f=x\left\{\left[(f, f)_{x} y(f * 1,]\right]_{y}+[(f x y)-f(x, y+1])^{\frac{1}{2}}\right]\right.
$$

If the gradient value $G[f(x, y)]$ is directly used to represent image, that's to say, make sure $\mathrm{g}(\mathrm{x}, \mathrm{y})=\mathrm{G}[\mathrm{F}(\mathrm{x}, \mathrm{y})$, it can be drawn that the value is small at the part where the image changes slowly (the image is dim), and large at the part the contour changes fast. That is the purpose of sharpening process to get the image clearer after gradient calculation. The calculation is simple and practical, while the trouble is that the intensified image only displays the margin contour with steep gay scale changes, and the part where the gray scale changes slowly or evenly appears in the black. Some boundaries with rare changes may perhaps break off, and the poor display of inner texture may also bring trouble to texture measurement. The calculation is perhaps suitable for the boundary test of binary image and some specific occasions.

Laplace sharpening is also inclined for the linear combination of the derivative calculation and the isotropic linear calculation,

The image $\mathrm{g}(\mathrm{i}, \mathrm{j})$ can be simplified and expressed like below after it is processed by Laplace sharpening from the image $f(i, j)$, based on its different sharpening coefficient.

$$
g(i, j)=5 f(i, j)-5 f(i-1, j)-5 f(i+1, j)-5 f(i, j+1)-f(i, j-1)
$$


it can be expressed in the pattern of template: $\left[\begin{array}{ccc}0 & -1 & 0 \\ -1 & 5 & -1 \\ 0 & -1 & 0\end{array}\right]$

The advantage of Laplace sharpening lies in isotropy (unchanged from rotation), and thus it is more suitable for the sharpening process for oval structure image. The calculation is straightforward for being possible to convert to template operation; while its disadvantage is the small affect on the parasite egg image of the loss of the boundary direction and the slight increase in noise.

\section{Calculation of parasite egg perimeter and size}

Perimeter and size measurement is generally the primary choice in microscopic image recognition. The circularity is generally the mathematical relationship between perimeter and size. They have different definition on different occasions of the literatures. Parasite eggs, for their irregular sections can be called shape complexity or shape complex factor, so in digital image process, perimeter and size are measured usually in polygon method and pixel method.

Polygon method defines irregular images as a polygon composed of many triangles with any point inside as their peaks. Its perimeter and size are calculated by measuring the total size of all the triangles and the total length of the base line. Generally it can be drawn by accumulating boundary traversal in boundary tracking.

Flood fill algorithm is conducted not by searching the boundary color value but by replacing the designated inside color and get the area painted. It is usually defined in this way:

Start from the designated inside point (x,y)paint the expected fill color over all the pixel set in designated color. Assume that the area to be painted has many inside colors; pixel value can be reset to get all the insides point the same color. Then use 4 or 8 -connected method to connect all the pixels step by step until all the inside points are painted. The source procedure is expressed like below, starting from the position of type-in to fill a 4-connected zone by recursion.

Flood fill algorithm has the following source procedure:

voidfloodFill4(intx,inty,intfillColor,intoldColor)

\{

if $($ getPixel $(\mathrm{x}, \mathrm{y})=$ "oldColor $)\{$

setColor(fillColor);

setPixel(x,y);

floodFill4( $\mathrm{x}+1, \mathrm{y}$, fillColor,oldColor):

floodFill4(x-1,y,fillColor,oldColor);

floodFill4(x,y+1,fillColor,oldColor);

floodFill4(x,y-1,fillColor,01dColor);

\}

Pixel method has the simplest principle - counting the number of the pixel points on the boundary (or inside the boundary). But due to the fact that the images of the parasite eggs has multiple boundary after binary cutting, the boundary lines are not even and nor the color inside the boundary, perimeter and size measurements have to face much trouble by pixel method and have to turn to a simple outer boundary search algorithm to find the outermost boundary; then, flood fill algorithm in computer graphics can be used for area painting, replace the boundary line with a 
pixel-wide boundary in the same color, and the inside of the boundary with another with even color, and respectively calculate the total pixels on and inside the boundary by scanning. That is how to easily calculate the perimeter and size of the parasite eggs.

\section{Texture analysis and calculation research on parasite eggs}

Texture analysis is developed from remote sensor image analysis technology [40]. With the development of satellite technology, it has been widely used in satellite cloud picture and typhoon recognition. In recent years, the wide application of human face, fingerprint and iris image recognition, especially the increasingly mature recognition method of fingerprints and iris, has pushed the texture analysis technology to a new height.

Texture analysis is a visual feature reflecting the homogeneity phenomenon in image not dependent on color and brightness. It is an inside characteristic on the surface of all things, serving as the major basis for human to recognize them.

Biological feature recognition is rated as one of the top ten high technologies in 21 st century. Bill Gates ever asserted that biological recognition technology, that's the technology using human physiological features like fingerprints and iris to recognize personal identity, will become an important reform in IT industry in future years. In the field of medical image process, Nanjing University of Science and technology and China Medical University ever made some progress in lung cancer cutting, reorganization and early diagnosis as early as in 1997; in February 2004, Sichuan University achieved $90.9 \%$ recognition accuracy by using grey scale co-occurrence matrix to analyze the physiological features from the B-ultrasonic images of the normal liver and fatty liver; and Denmark scientists also put worm egg texture features into the automatic recognition and research on three cattle nematodes eggs in 1998.

However, automatic recognition cannot work for all the worm eggs after normal pretreatment because of the disturbance of impurities, since some work eggs contains more impurities while some has a natural impurity absorption capacity, due to their different living environment and characteristics.

Based on image smoothing algorithm analysis, extra impurity removal process can be carried out for the images not very fine and the boundary not very thin, by use of simple linear low-pass filter (neighborhood average template) with a strong noise removal and blurring effects.

$$
\text { Template_SmpFilter }=\frac{1}{10}\left[\begin{array}{lll}
1 & 1 & 1 \\
1 & 2 & 1 \\
1 & 1 & 1
\end{array}\right]
$$

It can be expressed like below:

$$
\mathrm{gl}(\mathrm{m}, \mathrm{n})=\{2 \mathrm{f}(\mathrm{m}, \mathrm{n})+\mathrm{f}(\mathrm{m}-1, \mathrm{n})+\mathrm{f}(\mathrm{m}+1, \mathrm{n})+\mathrm{f}(\mathrm{m}, \mathrm{n}-1)+(\mathrm{fm}, \mathrm{n}+1) 1+[\mathrm{f}(\mathrm{m}-1, \mathrm{n}-1)+\mathrm{f}(\mathrm{m}-1, \mathrm{n}+1)+\mathrm{f}(\mathrm{m}+1, \mathrm{n}-1)+\mathrm{f}(\mathrm{m}+1,
$$
$\mathrm{n}+1)\} / 10$

Among $\mathrm{f}(\mathrm{m}, \mathrm{n})$ is the original image, $\mathrm{gl}(\mathrm{m}, \mathrm{n})$ is the image after smoothing.

Gaussian smoothing filter algorithm will not be specially discussed herein, since it has almost the same source procedure as the simple linear low-pass filter algorithm, except for the template elements.

By comparing the images of an unfertilized roundworm egg after impurity removal process respectively by normal process and simple linear low-pass filter, it can be known that simple linear 
low-pass filter can effectively achieve impurity removal effects when the normal process cannot work.

\section{Conclusions}

In combination with the theories on digital image process and model recognition, the recent progress of digital microscopic image recognition technology at home and abroad has been researched in the thesis, a complete description of the characteristics of the common human parasite egg microscopic image has been made, and some innovative results have also been achieved in the analytical research on the pretreatment, cutting, characteristics extraction and recognition algorithms suitable for various worm eggs.

(1) A comprehensive and in-depth research has been made on parasite eggs digital image recognition status and the problems at home and abroad, but there still lacks research overview in this regard at home, even when the topic is expanded to the field of the application of digital image technology in parasitology, the research documents can be more complete than what are current retrieved. Thus that has enhanced the starting point and the pertinence of the research.

(2) The research on the pretreatment and cutting algorithm of the worm eggs with impurities, complex shape and boundary can effectively overcome the disadvantaged factors and extract the useful parts of the image from its background and impurities to lay a sound foundation for follow-up character extraction and recognition. The analytical research method is helpful for similar research in the future.

(3) The characteristics of human parasite egg image have been comprehensively described, and their unique features have been accurately reflected and quantitatively measured with less characteristic number. There never appeared the classification disturbance phenomenon common in digital image recognition system in the process of recognition. The successful character selection and extraction can make sure the system realize quick recognition and classification for worm eggs in a simple classification algorithm.

(4) Multiple technologies have been used for careful research on worm egg image colors, and such conclusion is drawn as its color lacks the characters unrelated to image brightness. The effective application of color characters in classifying and recognizing worm eggs under the fixed optical parameters is also an useful exploration for the demarcation of optical parameters in future research and clinical application.

\section{References}

[1]Luo Shuqian, Zhou Hongguo. Medical Image Process and Analysis. Beijing: Science Press,2003

[2]He Lianyin, Yang Tiesheng. The Development of Laboratory Diagnosis of Parasitic Disease. Chin J Med Lab Sci,1999-01,22(1):15-18

[3]Zeng Xianfang. Parasitology and Parasitology Test. Beijing: People's Medical Publishing House, 2001,181-209

[4]Zeng Zhaofang. Clinical Examination Instrumentation. Beijing: People's Medical Publishing House, 2001,237-254 
[5]Li Shunyi. Morphological Observation Emphasizing on Routine Blood Tests. Laboratory Medicine Information Network/Online Database of Laboratory Medicine (http//www.elinet.eom.en/edu/resouree/artiele/20ollO19o4d.htm).2001

[6]Liu Bin, Zeng Libo, Liu Shenghao. Research on Blood Cell Image Automatic Identification System. Computer Engineering,2003-01,29(1):174-175

[7] Xi 'an Huahai Medical Information Technology Co., Ltd. Color Blood Cell Graphic Analysis System. :httP://www.Paes.eom.en/medPaes/1O.asP.2004-8-24

[8]Jiang Hongbing, Xia Yongxin, Wan Suiren, etc. Invention of Computer Microscopic Image Urinary Sediment Analyzer. Chinese Medical Equipment Journal,2001(4):6-8

[9]Thomas Wurflinger Wurflinger,Jens Stockhausen,Dietrieh Meyer-Ebreeht,et.al. Robust automatic coregistration,segmentation, and classifieation of cell nuclei in Multimodal cytoPathologieal microscopic images. Computerized Medieal Imaging and GraPhics.2004-01.28(1):87—98

[10] Lu Xinquan, Chen Shifu. Lung Carcinoma Cell Automatic Identification Classification System based on CP Machine, Computer System Application 1998(10):48 - 50 